\section{Efficacy of anti-PD1 re-treatment in patients with Hodgkin lymphoma who relapsed after anti-PD1 discontinuation}

Patients with relapsed/refractory Hodgkin lymphoma (R/R HL) experience high response rates upon anti-PD1 therapy. In these patients, there is limited data about the optimal duration of treatment and the risk of relapse after anti-PD1 discontinuation. We have previously reported the outcome of 11 patients with R/R HL who discontinued anti-PD1 therapy after achieving a complete response (CR) upon nivolumab. ${ }^{1}$ These patients experienced a favorable outcome as only two of them had relapsed after a median follow-up of 21.2 months from discontinuation. Despite the low relapse rate observed in that study, physicians may be worried about the possibility to further rescue these heavily pretreated patients in case of relapse after anti-PD1 discontinuation. Notably, it is still unknown whether these patients will remain sensitive to a second course of antiPD1.

Here, we investigated the efficacy of anti-PD1 retreatment in patients that were initially sensitive to anti-PD1 therapy but who relapsed after anti-PD1 discontinuation.

We retrospectively analyzed patients with $\mathrm{R} / \mathrm{R} H \mathrm{HL}$ who experienced a partial (PR) or CR upon anti-PD1 monotherapy and discontinued the treatment either because of unacceptable toxicity or due to prolonged remission, based on the physician's decision. Patients who discontinued anti-PD1 therapy because of relapse/progression or underwent consolidation with allogenic stem cell transplantation (alloSCT) were not included. Patients meeting the eligibility criteria were identified through the French lymphoma network (LYSA).

We identified seven patients who met the inclusion criteria. Their characteristics are summarized in Table 1. At anti-PD1 initiation, most patients presented with advanced disease (five of them had Ann Arbor stage III/IV) and had been heavily pre-treated (median number of prior systemic lines $=6$, seven had received prior brentuximab vedotin, five prior autologous stem cell transplantation (SCT) and two prior allogenic SCT). Overall, anti-PD1 was discontinued after a median duration of 11.4 months (range: $0-26.7$ ) and a median of 23 infusions (range: 1-51). Anti-PD1 was discontinued because of prolonged remission $(n=5)$ or toxicity $(n=2$, patient 5 had experienced grade 4 acute liver graft-versus-host-disease [aGvHD] and patient 7 grade 3 laryngeal tightness). The disease status at anti-PD1 discontinuation was CR for six patients and PR for one patient. The median time to relapse after anti-PD1 discontinuation was 12.1 months (range: 5.3-26.7). All patients were re-treated with the same anti-PD1 antibody as initially administered (six with nivolumab and one with pembrolizumab).

All patients responded to anti-PD1 re-treatment (Figure 1). The best response was CR for four patients and PR for three patients. At the time of analysis (median follow-up of 19.2 months from anti-PD1 re-treatment), 4 of 7 patients have ongoing responses to antiPD1 monotherapy, three of them beyond 12 months. Interestingly, three patients discontinued anti-PD1 treatment after achieving a second objective response upon anti-PD1 re-treatment (patients 3, 5 and 7). Patient 3 discontinued anti-PD1 treatment a second time due to hyper-eosinophilia and then relapsed 2
Table 1. Patients' characteristics at anti-PD1 initiation.

\begin{tabular}{|c|c|}
\hline Patients' characteristics & $N=7$ \\
\hline Age, median, years (range) & $47(34-71)$ \\
\hline Sex, male, number (\%) & $2(28.6)$ \\
\hline \multicolumn{2}{|l|}{ cHL subtype, number (\%) } \\
\hline Nodular sclerosis HL & $6(85.7)$ \\
\hline Unclassifiable & $1(14.3)$ \\
\hline \multicolumn{2}{|l|}{ Performance status (ECOG), № (\%) } \\
\hline $0-1$ & $5(71.4)$ \\
\hline$\geq 2$ & $2(28.6)$ \\
\hline \multicolumn{2}{|l|}{ Stage disease, number (\%) } \\
\hline $\mathrm{I} / \mathrm{II}$ & $2(28.6)$ \\
\hline III/IV & $5(71.4)$ \\
\hline Prior lines of systemic therapy, median (range) & $6(4-11)$ \\
\hline Prior radiation therapy, number (\%) & $4(57.1)$ \\
\hline Prior treatment with Brentuximab Vedotin, number $(\%$ & o) $7(100)$ \\
\hline Prior autologous HSCT, number (\%) & $5(71.4)$ \\
\hline Prior allogenic HSCT, number (\%) & $2(28.6)$ \\
\hline \multicolumn{2}{|l|}{ Anti-PD1, number (\%) } \\
\hline Nivolumab & $6(85.7)$ \\
\hline Pembrolizumab & $1(14.3)$ \\
\hline $\begin{array}{l}\text { Number of anti-PD1 infusions } \\
\text { during } 1^{\text {st }} \text { course, median (range) }\end{array}$ & $23(1-51)$ \\
\hline $\begin{array}{l}\text { Duration of } 1^{\text {st }} \text { course of anti-PD1 } \\
\text { therapy (months), median (range) }\end{array}$ & $11.4(0-26.7)$ \\
\hline \multicolumn{2}{|c|}{ Best Overall Response to $1^{\text {st }}$ course of anti-PD1, number (\%) } \\
\hline $\mathrm{CR}$ & $6(85.7)$ \\
\hline PR & $1(14.3)$ \\
\hline
\end{tabular}

Reason for anti-PD1 discontinuation, number (\%)

Prolonged response

Toxicity*

Disease status at anti-PD1 discontinuation, number (\%)

$\mathrm{CR}$

$6(85.7)$

PR

Time between discontinuation

$12.1(5.3-26.7)$

and relapse (months), median (range)

Follow-up from anti-PD1 re-treatment

$19.2(4.8-39.9)$

(months), median (range)

Best response to $2^{\text {nd }}$ course of anti-PD1

CR

PR

$3(42.9)$

* One grade 4 acute liver graft-versus-host-disease, and one grade 3 laryngeal tightness. HL: Hodgkin lymphoma; cHL: chronic Hodgkin lymphoma; CR: complete response; PR: partial response.

months later. Patient 7 tolerated the second course of nivolumab well (notably, there was no recurrence of laryngeal tightness), achieved a PR and discontinued the treatment after 12 months. Unfortunately, he relapsed 5 months later. The patient then received a third course of nivolumab and achieved another PR which is still ongoing at 18 months.

Patient 5 had undergone alloSCT prior to anti-PD1 therapy. He discontinued nivolumab after a single infusion due to grade 4 liver GvHD. Nevertheless, he achieved a CR which lasted 9 months. At relapse, he 


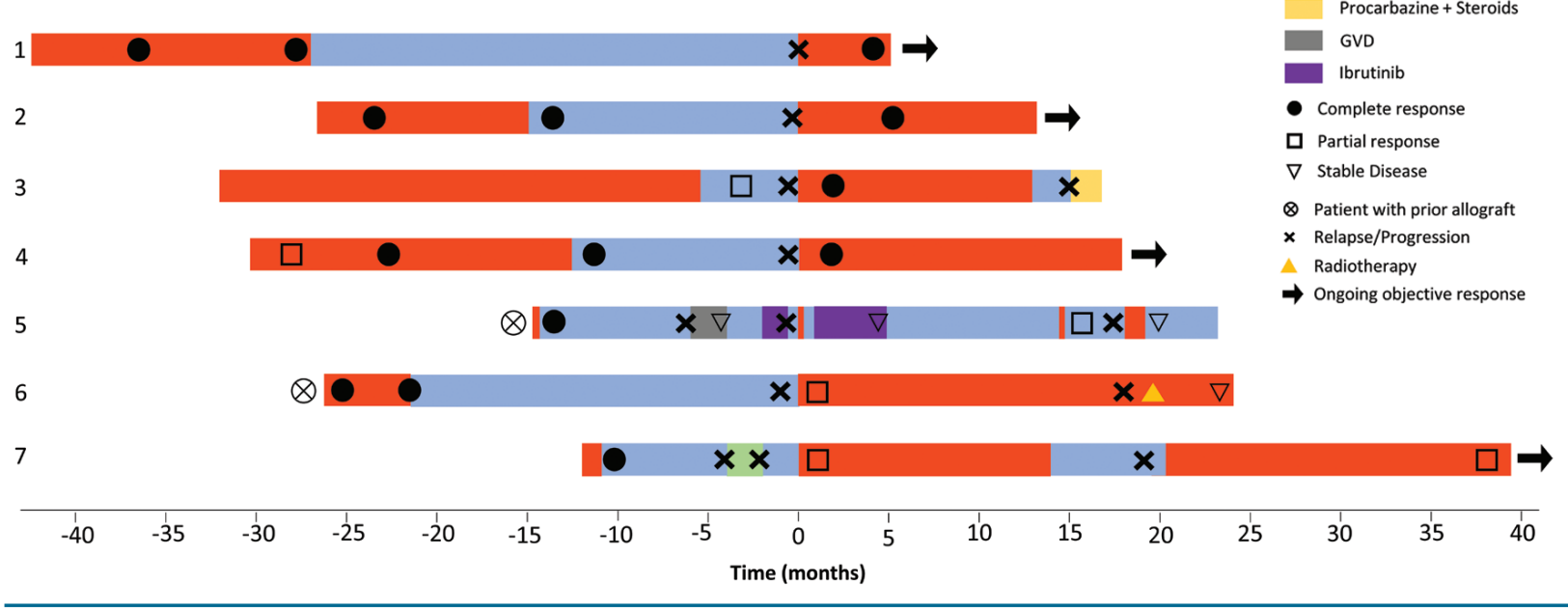

Figure 1. Efficacy of anti-PD1 re-treatment.

received a salvage therapy with GVD (gemcitabine, vinorelbine, and pegylated liposomal doxorubicin) followed by ibrutinib without efficacy. At progression, he received a reduced dose of nivolumab (total dose of 30 mg) which induced a GvHD flare. The patient further experienced two disease progressions which were treated with two additional infusions of low dose nivolumab (10 mg) which resulted again in GvHD flares. These sequential treatments induced transient lymphoma regression, with $\mathrm{PR}$ as the best response.

The GvHD flares observed in this patient are not unexpected. Indeed, prior studies have reported that anti-PD1 therapy potentially induces GvHD reactivation in patients that previously underwent alloSCT.,3 Anti-PD1 re-treatment did not induce other significant toxicities in the other patients.

There is only limited data regarding the efficacy of rechallenge with anti-PD1 antibodies in cancer patients. Only a few cases have been reported in patients with solid tumors, namely in non-small cell lung cancer ${ }^{4}$ and melanoma. ${ }^{5}$ However, most of these patients had failed initial anti-PD1 therapy and received chemotherapy prior to anti-PD1 re-treatment. This is in contrast to our study in which all patients were sensitive to initial antiPD1 therapy and were responsive (CR or PR) at the time of discontinuation.

At the ISHL11 meeting, Ansell et al. reported in an abstract the efficacy of anti-PD1 re-treatment in five patients who achieved remission upon nivolumab but relapsed after treatment discontinuation. ${ }^{4}$ All patients responded to nivolumab re-treatment (one $\mathrm{CR}$ and four $\mathrm{PR}){ }^{6}$ Recently, Chen et al. reported an update of the KEYNOTE-087 study after 2 years of follow-up. ${ }^{7}$ This study evaluated pembrolizumab in R/R HL. In a subset analysis, Chen et al. reported the outcome of 10 patients who received a second course of pembrolizumab after anti-PD1 discontinuation. Eight patients were evaluable and six of them $(75 \%)$ experienced an objective response upon re-treatment with pembrolizumab (four $\mathrm{CR}$ and two PR). The study neither reported on the efficacy of the first course of anti-PD1, the reasons for antiPD1 discontinuation nor the tumor status at anti-PD1 discontinuation in these patients. Nevertheless, the study shows that most patients responded to anti-PD1 re-treatment.

Our study, along with the studies recently published by Ansell et al. ${ }^{6}$ and Chen et al., ${ }^{7}$ are the first to report on the efficacy of anti-PD1 re-treatment in anti-PD1 sensitive, R/R HL patients who relapsed/progressed after anti-PD1 discontinuation. These two studies show high response rates $(75-100 \%)$ after anti-PD1 re-treatment suggesting that these patients usually remain "anti-PD1 sensitive" at relapse. These observations suggest that anti-PD1 is an effective salvage therapy for HL patients who relapse after anti-PD1 discontinuation. Larger studies are warranted to confirm these results.

Guillaume Manson, ${ }^{1}$ Pauline Brice, ${ }^{2}$ Charles Herbaux, ${ }^{3}$ Kamal Bouabdallah, ${ }^{4}$ Chloé Antier, ${ }^{5}$ Florence Poizeau, ${ }^{6,7}$ Laurent Dercle ${ }^{8-10}$ and Roch Houot ${ }^{1}$

${ }^{2}$ Department of Hematology, University Hospital of Rennes, Rennes, France; 'Department of Hematology, Saint-Louis Hospital, AP-HP, Paris, France; ${ }^{3}$ Department of Hematology, University Hospital of Lille, Lille, France; ${ }^{4}$ Department of Hematology, University Hospital of Bordeaux, Bordeaux, France; ${ }^{5}$ Department of Hematology, Nantes University Hospital, Nantes, France; ${ }^{6}$ Department of Dermatology, University hospital of Rennes, Rennes, France; 'EA 7449 REPERES

(Pharmacoepidemiology and Health Services Research), Rennes 1 University, Rennes, France; ${ }^{8}$ Medical Imaging Department, Institut Gustave Roussy, Villejuif, France; ' UMR1015, Institut Gustave Roussy, Villejuif, France and ${ }^{10}$ Department of Radiology, Columbia University Medical Center, New York, NY, USA

Correspondence:

ROCH HOUOT - roch.houot@chu-rennes.fr

doi:10.3324/haematol.2019.242529

\section{References}

1. Manson G, Herbaux C, Brice P, et al. Prolonged remissions after anti-PD-1 discontinuation in patients with Hodgkin lymphoma. Blood. 2018;131(25):2856-2859.

2. Haverkos BM, Abbott D, Hamadani M, et al. PD-1 blockade for relapsed lymphoma post-allogeneic hematopoietic cell transplant: high response rate but frequent GVHD. Blood. 
2017;130(2):221-228

3. Herbaux C, Gauthier J, Brice P, et al. Efficacy and tolerability of nivolumab after allogeneic transplantation for relapsed Hodgkin lymphoma. Blood. 2017;129(18):2471-2478

4. Fujita K, Uchida N, Kanai O, Okamura M, Nakatani K, Mio T Retreatment with pembrolizumab in advanced non-small cell lung cancer patients previously treated with nivolumab: emerging reports of 12 cases. Cancer Chemother Pharmacol. 2018;81(6):1105-1109.

5. Nomura M, Otsuka A, Kondo T, et al. Efficacy and safety of retreatment with nivolumab in metastatic melanoma patients previously treated with nivolumab. Cancer Chemother Pharmacol. 2017;80(5):999-1004.

6. Ansell SM, Armand P, Timmerman JM, et al. Nivolumab re-treatment in patients with relapsed/refractory Hodgkin lymphoma. Presented at the $11^{\text {th }}$ International Symposium on Hodgkin Lymphoma. Abstract \#0116, 2018 Cologne, Germany

7. Chen R, Zinzani PL, Lee HJ, et al. Pembrolizumab in relapsed or refractory Hodgkin lymphoma: two-year follow-up of KEYNOTE-087. Blood. 2019;134(14):114-1153. 